\title{
Positive Effect of the Use of Accelerometry on Lifestyle Awareness of Overweight
}

\author{
Hypertensive Patients
}

Laura Stefani $^{* 1 \mathrm{AD}}$, MD; Gabriele Mascherini ${ }^{1 \mathrm{BC}}$, PhD; Irene Scacciati ${ }^{1 \mathrm{BC}}$; Alessio De Luca $^{\text {1BC }}$; Nicola Maffulli ${ }^{2 D E}$, MD, PhD, FRCS(Orth); Giorgio Galanti ${ }^{1 D E}$, MD

\begin{abstract}
Purpose: Sedentary habits are strongly associated with increase of cardiovascular risk factors. The present study aimed to verify the role of accelerometry in identifying sedentary behavior, and the possible short term positive effect of this intervention on some anthropometric variables in a group of patients with cardiovascular risks factors.
\end{abstract}

Methods: To quantify daily Spontaneous Motor Activity (SMA) levels and identify sedentary behavior, an accelerometer was employed, in addition to a simple questionnaire, in a group of overweight, hypertensive subjects. A personalized unsupervised 3 month long physical exercise program was planned. After this time, acceleroometry was again undertaken to analyze the impact of the intervention on some life style parameters, including the number of the steps taken daily, and Physical Activity Level (PAL). In addition, body water balance, weight, and Body Mass Index (BMI) were also evaluated.

Results: Assessment of physical activity by accelerometry identifies sedentary behaviors in a larger number of individuals. After three months of regular unsupervised aerobic exercise, PAL improved from $1.56 \pm 0.1$ to $1.68 \pm 0.2$ with $P<0.005$, weight $(\mathrm{kg})$ reduced from $85.13 \pm 20$ to $83.10 \pm 19(P<0.05)$, BMI from 29.58 to $28.7(P<0.05)$.

Conclusion: Accelerometry allows to objectify PAL, and can be used to monitor improvement of variables strongly related to cardiovascular risk.

Key Words: Lifestyle; Accelerometer; Exercise as Prescription Therapy; Body Composition

Asian Journal of Sports Medicine, Volume 4 (Number 4), December 2013, Pages: 241-248

\section{INTRODUCTION}

$\mathrm{T}^{\mathrm{h}}$ he complete definition of lifestyle normally includes all those behaviors closely related to the nutritional and physical activity aspects which are aimed at maintaining wellness ${ }^{[1]}$. Sedentarism represents one of the crucial points of unhealthy lifestyle. Correct lifestyle habits are associated with progressive reduction of acute cardiovascular events with an increase in survival ${ }^{[2]}$. A healthy lifestyle can also reduce costs in terms of use of medications and social support ${ }^{[3,4]}$. More recently, the use of wearable systems to measure physical activity has been progressively advocated ${ }^{[5,6]}$ to avoid sedentarism. Usually, daily evaluation of lifestyle is the first step to start with "Exercise as prescription". Particularly in subjects at high cardiovascular risk, the correct identification of daily Spontaneous Physical Activity (SPA) can be used to plan exercise prescription ${ }^{[7,8]}$. Therefore, for exercise to play a role in the management of chronic conditions, a valid outcome to measure physical activity is at the same time essential in reducing the development of chronic disease and to delay premature mortality ${ }^{[9]}$. The literature supports the use of accelerometry instead of simple questionnaires. The latter may contain subjective 
elements which lead to imperfect estimation of the daily habits. This aspect is particularly relevant if the exercise proposed is unsupervised or in case of older people in whom wearable motion sensing technology can be safely employed to study movement ${ }^{[10,11]}$. However, the clinical application and the possible immediate effects after a short period of use to assess SPA in chronic diseases has not been studied. The present investigation aims to verify the clinical relevance and feasibility of applying wearable motion sensing technology in individuals at high cardiovascular risk. The principal aim of the study is to highlight the role of accelerometry in objectifying sedentary behaviors in a group of subjects with cardiovascular risks factors. The study was designed as a 'before and after' observational investigation comparing physical activity levels before and after a period of unsupervised physical exercise in the subjects who used a portable accelerometer 1 .

\section{METHODS AND SUBJECTS}

A cohort of 28 hypertensive overweight patients (14 male, 14 female aged $61.23 \pm 11.30 \mathrm{y}$ ) were enrolled in an exercise prescription program planned at the Sport Medicine Center at the University of Florence, Italy. All subjects gave their informed consent to participate in the study. All subjects had grade I-II hypertension, according to the ESC-AHA classification ${ }^{[12]}$, under treatment with ACE-inhibitor or calcium-antagonist drugs. At the onset of the study, all subjects underwent a detailed clinical examination and echocardiography to verify the absence of other cardiovascular conditions as a possible contraindication for regular physical exercise. At echocardiography, systolic and diastolic left ventricle (LV) parameters were measured ${ }^{[13]}$. Echocardiography was conducted by two experienced certified cardiologists using the ESAOTE My Lab 50 echocardiograph equipped with a $2.5 \mathrm{MHz}$ probe. Each subject was examined using mono-bidimensional echocardiographic and Doppler tests. According to the American Society of Echocardiography guidelines, end-systolic and end-diastolic left ventricle diameters (LVEDd, LVESD), interventricular septum (IVS) and posterior wall (PW) thickness, left atrium (LA) and aorta (Ao) root starting from the long parasternal axis view were measured ${ }^{[14]}$. The LV Mass Index (LVMI) was calculated following Devereux's formula [15] Considering the regularity of the geometry of the left ventricular chamber of the subjects, the ejection fraction (EF \%) was calculated according to the formula (LVEDd - LVESd / LVEDd), for which volumes are substituted by diameters. The analysis of the diastolic variables was performed in the presence of a stable RR interval and in three different but sequential measurements from the 4 chamber view consisting of the measurements, by Doppler analysis, of transmitral flow of $\mathrm{E}$ wave and $\mathrm{A}$ wave peak velocities, isovolumetric relaxation and deceleration times and E/A ratio from 4 chambers view. The formula weight $(\mathrm{kg}) /$ height $(\mathrm{m})^{2}$ was applied to calculate the body mass index (BMI). The lifestyle self perception of the subjects studied was investigated by a questionnaire. The questionnaire used was extracted from a validated questionnaire reported in the ACSM guidelines ${ }^{[16]}$, and translated into Italian. It consisted of a one to one interview with each subject enrolled in the study during the initial clinical examination, before the start of the exercise program. The questionnaire collected data about occupation, leisure time activities (watching TV, reading, cycling, planning indoor games), smoking behavior, alcohol intake, physical activity and dietary habits. From the questionnaire data, spontaneous physical activity evaluation or programmed daily exercise such as fast walk or jogging were verified. Among the variables considered, the frequency (i.e. once, twice, three or more times a week) and the duration (expressed in minutes) of physical activity were considered to estimate lifestyle behavior, and expressed in METs ${ }^{[17]}$. From the information obtained, the subjects were therefore classified as "sedentary" or "active". Subjects were considered sedentary if the amount of daily physical activity was lower than 2 METs, and active if the amount of daily physical activity was 3-4 METs. Assessment of lifestyle was completed using a commercially available accelerometer (AiperMotion 500 PC- Aipermon GmBH -Germany). The 
accelerometer was positioned on the waist of the patient, asking the subject to wear the device for 6 days except at night. After this period, the data obtained from the accelerometer were downloaded to a laptop computer to analyze SPA: more than 3 hours a week represented the threshold point to distinguish sedentary from active subjects. Physical Activity Level (PAL), which derives from the ratio of the total energy expenditure (TEE) divided by the subject's own basal metabolic rate (BMR), was also calculated. BMR was estimated using the formula by Harris and Benedict on the basis of weight, height and age of the patient ${ }^{[18]}$. These two variables are calculated directly from the accelerometer report over a 24 hour period. The mean value of PAL as cut-off level to distinguish sedentary from active subjects is normally established at $1.6^{[19]}$. From the report of the accelerometer, the daily PA is further divided into "slow walking " $(\mathrm{LW})$, "fast walking" (FW) and jogging. In addition, the mean daily distance and the intensity of the PA walk (expressed as slow walking up to $3 \mathrm{Km} / \mathrm{h}$ or fast walking up to $5 / \mathrm{km} / \mathrm{h}$ ), and also the total number of daily steps were obtained.

Following the AHA ACC guidelines, the amount of physical exercise prescribed was established from a Cardio Pulmonary Exercise Test (CPET) [20] performed at the onset of the protocol to estimate Energy Expenditure (EE).

The CPET was performed on a treadmill (Schiller Cardiovit Ergo-Spiro CS 200 treadmill, Schiller AG, Baar, Switzerland). During the test, the slope and velocity of the ramp were increased according the modified Bruce protocol. The test analyzes the respiratory gas measured during the exercise. The transducer with mouthpiece detects, breath by breath, oxygen uptake (VO2), the expired $\mathrm{CO} 2$ (VCO2), and minute ventilation (VE). The EE calculation, after the end of the test and the individuation of the Aerobic Threshold (AerT) and Anaerobic Threshold (AT). The measurement of AT was undertaken using three different methods: v-slope method, ventilatory equivalent and the respiratory quotient.

The v-slope method identifies the AT as the VO2 at which the change in slope of the relationship of $\mathrm{VCO} 2$ to $\mathrm{VO} 2$ occurs. With the ventilatory equivalent, the AT is defined as the $\mathrm{VO} 2$ at which $\mathrm{VE} / \mathrm{VO} 2$ and
Pulmonary end-tidal $\mathrm{O} 2$ (PetO2) reach a minimum and thereafter begin to rise consistently, coinciding with an unchanged VE/VCO2 and Pulmonary end-tidal $\mathrm{CO} 2$ (PetCO2). The respiratory quotient method identifies $\mathrm{AT}$ as the VO2 at the time when the respiratory exchange ratio stabilized above 1.0 and does not return to below 1.0. The AT values were obtained during the CPT by v-slope method: this method identifies AT as the $\mathrm{VO} 2$ point (where there is the change in slope of the relationship of $\mathrm{VCO} 2$ to VO2) [21]. To clarify the intensity of the physical exercise, an additional method was used. Before starting with the CPT, the CR 10 scale [22] was shown to the subjects enrolled, explaining to them the relationship between the degree of the scale and the intensity of the effort. Subjects were invited to identify the intensity of the effort showing a number using their fingers just before the end of the maximal effort performed during the CPT. This aspect was used in our investigation as an educational tool to easy adhere to the exercise program. The RPE (Rate of Perceived Exertion) corresponds almost precisely to a particular intensity of the effort $[23,24]$ and offers the subjects the opportunity to better modulate their effort during their physical training program, especially when it is unsupervised.

The EE measurement was possible from the calculation of the corresponding METs at the AerT (aerobic threshold). The EE index quantifies the total amount of physical activity performed in a standardized manner across individuals and types of activities, calculated as the product of the number of METs associated with one or more physical activities and the number of minutes of the activities (i.e. METs for minutes) from the formula: $\mathrm{EE}=(\mathrm{METs} \times \mathrm{Kg}) / 60$ where: $\mathrm{EE}=$ Energy Expenditure; METs = METs corresponding at the maximum intensity prescribed (METs metabolic Equivalent); $\mathrm{kg}=$ Kilogram; $60=$ minutes per hour ${ }^{[20]}$.

The duration of exercise has been established by the formula Time of exercise $=150 * * / \mathrm{EE}$.

**150 represents the time currently recognized and recommended for the weekly exercise as prescription therapy.

The intensity of one's individual exercise was set at the heart rate between the aerobic (AerT) and the anaerobic threshold (AT), at around $60 \%$ of $\mathrm{VO} 2$ peak for at least 3 times in a week. To guarantee optimal 
adherence to the physical exercise program prescribed, subjects were invited to use a wearable heart rate monitor to reach and maintain the range of HR suggested, or, if this was not possible, to continue the exercise close to the intensity felt during the application of the RPE scale for the appointed time. The exercise prescribed consisted of aerobic exercise such as fast walking or slow jogging. Bioelectrical Impedance Analysis (BIA) (Bioelectrical Impedance Analysis-Akern/STA/BIA -Italy) was used to measure water body composition at the beginning (T0) and after maintenance of normal heart function. All data are expressed as mean \pm standard deviation (SD), and were compared at the beginning and at the end of the study using the Student $t$-test for paired data. A probability value $(P)$ of $<0.05$ was considered statistically significant.

\section{RESULTS}

At the onset of the study, all the subjects were overweight, with BMI values between 25 and 30 . Using the questionnaire, 10 of 28 subjects were classified as sedentary (Table 1), while accelerometry at the same time (T0) showed a predominantly inactive lifestyle (Table 2), with a mean PAL value of $1.56 \pm 0.1$. The mean daily distance covered was $6289.3 \pm 2536.1$ $\mathrm{m}$, and the mean number of daily steps was $9522.4 \pm 3987.1$. The mean daily calories used were $878.6 \pm 302.1$ (Table 2). The mean amount of inactivity time during 6 days was $51 \pm 7.48$ hours, while the mean hours of exercise corresponding to 3 METs were $21.9 \pm 6.22$, the mean time of exercise at 3-4.5 METs was $10.88 \pm 4.11$ hours, and the mean time spent jogging was $73.54 \pm 8.66$ minutes. The sedentary subjects spent $8 \mathrm{~h} 30 \mathrm{~min}$ each day being sedentary, $3 \mathrm{~h}$ $45 \mathrm{~min}$ for slow walking, and $1 \mathrm{~h} 50 \mathrm{~min}$ for fast walking.

\section{the end of the observational period, a new} echocardiographic exam was performed to verify the
After three months of exercise all the subjects were revaluated. The anthropometric and the accelerometry

parameters were assessed again at three months). At setting using a manual blood pressure monitor. Statistical analysis was performed using the SPSS 20 package for Windows 7. chocardiographic exam was performed to verify the

Table 1: Questionnaire investigation, Report of the questionnaire investigation among the overweight-hypertensive subjects

\begin{tabular}{|llc|}
\hline Question & Description & No of subject \\
\hline \multirow{3}{*}{ Activity Self Perception } & Sedentary & 10 \\
& Moderate Active & 12 \\
& Active & 6 \\
& Pensioners & 10 \\
Occupational Activity & Housewife & 6 \\
& Sedentary Work & 7 \\
& Active Work & 5 \\
& Watching TV / Reading & 12 \\
& Modeling & 2 \\
& Gardening & 4 \\
& Walking & 6 \\
& Gymnastics & 1 \\
& Cycling & 2 \\
& Evening Sport & 1
\end{tabular}


Table 2: Physical Activity Level expressed as daily and weekly duration time

$\begin{array}{lccc}\text { Physical Activity } & \text { T 0 } & \text { T 3 } & \text { P. value } \\ \text { Physical Activity Level } & 1.56(0.1) & 1.68(0.2) & <0.05 \\ \text { Daily Distance (m) } & 6289.3(2536.2) & 7035.7(3237.3) & <0.05 \\ \text { Daily Steps } & 9522.4(3987.1) & 10824.1(4980.4) & <0.05 \\ \text { Daily Calories Spent (Kcal) } & 878.6(302.1) & 930.8(157.5) & \text { N.S }\end{array}$

NS: Non-significant

All the values obtained from the CPT were within normal ranges: $\mathrm{VO} 2$ peak was $23.3 \pm 3.3 \mathrm{mlO} 2 / \mathrm{kg} / \mathrm{min}$ with HR of $150.5 \pm 8.7 \mathrm{bpm}$; Anaerobic Threshold $18.9 \pm 2.9 \mathrm{mlO} 2 / \mathrm{kg} / \mathrm{min}$ and $\mathrm{HR} 117.3 \pm 6.6 \mathrm{bpm}$; Aerobic Threshold $10.6 \pm 2.3 \mathrm{mlO} / \mathrm{kg} / \mathrm{min}$ and HR $98.1 \pm 4.5$ bpm.

During the 3 months of the intervention, none of the subjects reported cardiovascular symptoms, and therefore none of them was excluded from the study, and none altered their pharmacological antihypertensive therapy.

After three months (T3) of regular exercise, sedentary behavior was newly evaluated using the wearable accelerometry for 6 days. The data obtained showed a significant increase in global physical activity expressed by an increase in the daily steps and also in PAL (Table 2).

BMI was significantly reduced $(\mathrm{T} 0=29.58 \pm 8.33$; $\mathrm{T} 3=28.7 \pm 8.75 \mathrm{P}<0.05)$. Although a reduction was observed also for the $\mathrm{FM}(\mathrm{T} 0=25.88 \pm 10.6$; $\mathrm{T} 3=$ $23.11 \pm 7.98)$, the $\mathrm{WC}(\mathrm{T} 0=101 \pm 14 ; \mathrm{T} 3=94.66 \pm 14)$, the HC $(\mathrm{T} 0=107 \pm 6.88 ; \mathrm{T} 3=100.9 \pm 2)$ the $\mathrm{TBW}(\mathrm{T} 0=$ $48.9 \pm 8.9 ; \mathrm{T} 3=41.24 \pm 9.55)$ and $\mathrm{ICW}(\mathrm{T} 0=24.01 \pm 6.32$; $\mathrm{T} 3=22.92 \pm 6.13$ ) (Table 3 ). This was not statistically significant. This was also the case for the mean systolic and diastolic blood pressure (T0= SBP:129.65 \pm 12.02
$\mathrm{mmHg} / \quad$ DBP: $78.11 \pm 7.35 \mathrm{mmHg} ; \quad \mathrm{T} 3=\mathrm{SBP}:$ $123.23 \pm 9.66 \mathrm{mmHg}$ / DBP: $73.57 \pm 4.88 \mathrm{mmHg}$ ). The AP values remained within the normal range $(\mathrm{T} 0=$ $7.55 \pm 1.6 ; \mathrm{T} 3=6.67 \pm 1.24)$ after the exercise period. Evidence of a negative association between PAL and the BMI values was found $(\mathrm{R}=-0.31)$.

Changes of echocardiographic parameters at the beginning and after 3 months of exercise were not statistically significant (Table 4).

\section{DISCUSSION}

Exercise is part of a healthy lifestyle, and adequate "Exercise as Prescription program" is normally associated with a reduction of the global cardiovascular risk ${ }^{[25,26]}$. Several difficulties are however present to evaluate the kind of physical activities practised, and a possible predominant sedentary behavior is not easy to demonstrate ${ }^{[26]}$. Normally, if only a "questionnaire" [27] is used for this purpose, an erroneous and incomplete evaluation ${ }^{[28]}$ of one's own lifestyle is possible regarding the type and the intensity of the exercises practised. Also, there is a tendency to underestimate

Table 3: Anthropometrics and body composition parameters of the subjects enrolled

$\begin{array}{lccc}\text { 28 subjects } & \text { T 0 } & \text { T 3 } & \text { P value } \\ \text { Weight (kg) } & 85.1(20.1) & 83.0(19.5) & <0.05 \\ \text { Body Mass Index } & 29.6(8.3) & 28.7(8.7) & <0.05 \\ \text { Fat Mass (kg) } & 25.9(10.6) & 23.1(8.0) & \text { NS } \\ \text { Waist Circunference (cm) } & 101.0(14.0) & 94.7(14.0) & \text { NS } \\ \text { Hip Circunference (cm) } & 107.0(6.9) & 100.9(2.0) & \text { NS } \\ \text { Total Body Water (l) } & 48.9(8.9) & 41.2(9.5) & \text { NS } \\ \text { Intra Cellular Water (l) } & 24.0(6.3) & 22.9(6.1) & \text { NS } \\ \text { Angle Phase (degrees) } & 7.5(1.6) & 6.7(1.2) & \text { NS } \\ \text { Systolic blood pressure (mmHg) } & 129.6(12.0) & 123.2(9.7) & \text { NS } \\ \text { Diastolic blood pressure (mmHg) } & 78.1(7.3) & 73.6(4.9) & \text { NS }\end{array}$

Trend toward a reduction of the main anthropometrics and body composition parameters (NS: Non-significant) 
Table 4: Echocardiographic parameters at the beginning (T0) and after 3 months of exercise (T3)

\begin{tabular}{|c|c|c|c|}
\hline Parameter & T0 & T3 & $P$. value \\
\hline Aortic root (mm) & $31.52(3.9)$ & $32.69(3.59)$ & NS \\
\hline Left Atrium (mm) & $38.70(5.6)$ & $40.69(4.76)$ & NS \\
\hline Inter Ventricle Septum (mm) & $9.74(0.7)$ & $9.79(0.6)$ & NS \\
\hline Posterior Wall (mm) & $9.78(0.9)$ & $9.69(0.7)$ & NS \\
\hline Left Ventricle End-Diastolic diameter (mm) & $47.76(3.7)$ & $48.92(3.2)$ & NS \\
\hline Left Ventricle End- Systolic diameter (mm) & $30.29(3.2)$ & $30.69(4.5)$ & NS \\
\hline Cardiac Mass Index (gr/m²) & $104.41(19.4)$ & $106.46(17.3)$ & NS \\
\hline Ejection Fraction \% & $63.41(3)$ & $62.92(3.1)$ & NS \\
\hline E wave (msec) & $69.11(36.3)$ & $73.07(39.9)$ & NS \\
\hline A wave (msec) & $62.52(1.8)$ & $69.61(17.5)$ & NS \\
\hline Decelation Time (ms) & $154.92(23.03)$ & $201.46(23.6)$ & NS \\
\hline Isovolumic Relaxation Time (ms) & $81.82(80.0)$ & $83.23(8.7)$ & NS \\
\hline Right Ventricle (mm) & $23.58(1.2)$ & $24.36(0.9)$ & NS \\
\hline
\end{tabular}

NS: Non-significant

the PAL often associated with high BMI values ${ }^{[29]}$.

More recently, the use of accelerometry has grown in both health and disease ${ }^{[30]}$. The data obtained in the present study support the role of accelerometry in identifying, more reliably than with a questionnaire, the presence of the "sedentary behaviours" ${ }^{[31]}$. In this way, individuals can be given objective quantification of their activities, and may take measures to increase the amount of exercise to improve their lifestyle and reduce their BMI ${ }^{[32]}$.

Our data support the educational role of accelerometry, which could be used as an additional tool for the periodical assessment of lifestyle changes to enhance adhesion to physical exercise prescriptions. We are aware of the fact that our study is limited by the number of subjects enrolled: this should prompt larger studies. The hemodynamic parameters remained essentially unchanged after three months of exercise. However, there were significant changes in both BMI and levels of physical activity in this period ${ }^{[33]}$. In this respect, our data should be considered preliminary, though encouraging, and should form the basis for larger, more extensive and longer, possibly randomised, studies on the effects of exercise prescription on mildly hypertensive overweight previously sedentary subjects. The introduction of exercise prescription would also decrease the use of anti-hypertensive medications, and thus have marked medico-economic benefits.

The identification of the presence of a predominant moderate PAL in a high risk group can therefore play a role to encourage to start with a "more adequate" PA program where the subjects work within a balanced program from moderate to vigorous physical exercise according to the ACSM Guidelines ${ }^{[16,34]}$. Previous studies underlined the relevance of the positive effects of the use of accelerometry as a tool to measure physical activity in several other chronic conditions such as arthritis or diabetes ${ }^{[35,36]}$, with a progressive increase of daily activity ${ }^{[35]}$. The use of unobtrusive accelerometers could be an additional system for periodical life style evaluation.

Accelerometry can verify effective spontaneous physical activity and quantify the time spent to practice exercise at different intensity levels ${ }^{[5,30]}$. The lack of recognition of physical inactivity is a barrier to changes in health behavior ${ }^{[2]}$. Particularly in ambulatory patients in whom we wish to reduce the cardiovascular risks, accelerometers can be proposed to better visualize the effective exposure to sedentary lifestyle, and give patients immediate feedback regarding their own lifestyle ${ }^{[29,32]}$.

\section{CONCLUSION}

Accelerometry allows to objectify PAL, and can be used to monitor improvement of variables strongly related to cardiovascular risk. 


\section{ACKNOWLEDGMENTS}

We declare that we have no conflict of interest and no funding has been received for this manuscript. The authors declare they have received the permission from the subjects analyzed for the present study.

Conflict of interests: None

\section{REFERENCES}

[1] Shrestha P, Ghimire L. A review about the effect of life style modification on diabetes and quality of life. Glob J Health Sci 2012;4: 185-90.

[2] Helajärvi H, Pahkala K, Raitakari O, et al. Reduced sitting time improves health. Duodecim. 2013;129(1):51-6.

[3] Hemmings S, Conner A, Maffulli N, et al. Cardiovascular disease risk factors in adolescent British South Asians and whites: a pilot study. Postgrad Med 2011;123:104-11.

[4] Lin BA, Thomas P, Spiezia F, et al. Changes in daily physical activity before and after total hip arthroplasty. A pilot study using accelerometry. Surgeon 2013;11:87-91.

[5] Bravata DM, Spangler CS, Sundaram V, et al. Using pedometers to increase physical activity and improve health: a systematic review. JAMA 2007;298:2296-304

[6] Tudor-Locke C, Basset DR. How many steps /day are enough? Preliminary pedometers indices, for public health. Sports Med 2004;34:1-8.

[7] Myers AM, Holliday PJ, Harvey KA, et al. Functional Performance Measures: Are They Superior to Self-Assessments? J Gerontol 1993;48:196-206

[8] I-Min Lee. Physical activity and cardiac Protection. Exercise is Medicine. 2010;9:214-9.

[9] Beaglehole R, Ebrahim S, Reddy S, et al. Prevention of Chronic Diseases: A Call to Action. Lancet 2007; 370:2152-7.

[10] Yach D, Hawkes C, Gould C.L, et al. The Global Burden of Chronic Diseases: Overcoming Impediments to Prevention and Control. JAMA 2004;291:2616-22.

[11] De Bruin ED, Hartmann A, Uebelhart D, et al. Wearable Systems for Monitoring Mobility-Related Activities in Older People: A Systematic Review. Clin Rehabil 2008; 22:878-895.

[12] Mancia G, De Backer G. The Task Force for the Management of Arterial Hypertension of the European Society of Hypertension (ESH) and of the European Society of Cardiology (ESC). Eur Heart J 2007;28:462-1536.

[13] Calderón FJ, Díaz V, Peinado AB, et al. Cardiac dimensions over 5 years in highly trained long-distance runners and sprinters. Phys Sportsmed 2010;38:112-8.

[14] Thomas JD. Appropriate use criteria in echocardiography: delphic or not? JASE 2011;24:268-70.

[15] Devereux RB. Detection of left ventricular hypertrophy by M-mode echocardiography. Anatomic validation, standardization and comparison to other methods. Hypertension 1987;20:1251-60.

[16] Thompson WR. ACSM Guidelines for Exercise Testing and Prescription. Eighth Edition, Lippincott Williams \& Wilkins 2010.

[17] McArdle, WD, Katch, FI, Katch VL. Exercise Physiology: Energy, Nutrition and Human Performance. Baltimore: Williams \& Wilkins 1996

[18] Roza AM, Shizgal HM. The Harris Benedict equation reevaluated: resting energy requirements and the body cell mass. Am J Clin Nutr 1984;40:168-82.

[19] Richardson CR, Newton TL, Abraham JJ, et al. A meta-analysis of pedometer-based walking interventions and weight loss. Ann Fam Med 2008;6:69-77.

[20] Gibbons RJ, Elliott M, Antman. ACC/AHA 2002 Guideline Update for Exercise Testing. A Report of the American College of Cardiology/American Heart Association Task Force on Practice Guidelines (Committee on Exercise Testing). Circulation 2002;106:1883-92

[21] Beaver WL, Wasserman K, Whipp BJ. A New Method for Detecting Anaerobic Threshold by Gas Exchange. J Appl Physiol 1986;60:2020-7.

[22] Scherr J, Wolfarth B, Christle JW, et al. Association between Borg's rating of perceived exertion and physiological measures of exercise intensity. Eur J Appl Physiol 2013;113:147-55.

[23] Coquart JB, Tourny-Chollet C, Lemaitre C, et al. Relevance of the measure of perceived exertion for the rehabilitation of obese patients. Ann Physical Rehabilitation Med 2012;55:623-40.

[24] Jakicic JM, Donnelly JE, Pronk NP et al. Prescription of exercise intensity for the obese patient: the relationship between heart rate, VO2 and perceived exertio. Int J Obes Relat Metab Disord 1995;19:382-7.

[25] Brown A, Siahpush M. Risk factors for overweight and obesity: results from the 2001 National Health Survey. Public Health 2007;121:603-13. 
[26] Garber CE, Blissmer B, Deschenes MR, et al. Quantity and Quality of Exercise for Developing and Maintaining Cardiorespiratory, Musculoskeletal, and Neuromotor Fitness in Apparently Healthy Adults: Guidance for Prescribing Exercise. position stand. Med Sci Sports Exerc 2011;43:1334-59.

[27] Pereira MA, FitzGerald SJ, Gregg EW, et al. A collection of Physical Activity Questionnaires for health-related research. Med Sci Sports Exerc 1997;29:S1-205.

[28] Historical leisure activity questionnaire. Med Sci Sport Exerc 1997;29:43-5.

[29] Ronda G, Van Asserrna P, Brug J. Stages of change, psychological factors and awareness of physical activity levels in the Netherlands. Health Promot Int 2001;16:305-314.

[30] Bassett DR, Wyatt H, Thompson H, et al. Pedometer-measured physical activity and health behaviors in US adults. Med Sci Sport Exerc 2010;42:1819-25.

[31] Melanson EL, Knoll JR, Bell ML. Commercially available pedometers: consideration for accurate step counting. Prev Med 2004; 39:361-8.

[32] Tudor-Locke CE, Ainsworth BE, Whitt MC, et al. The relationship between pedometer- determined ambulatory activity and body composition variables. Int J Obesity 2001;25:1571-8.

[33] Lee D, Sui X, Enrique G, et al. Long-Term Effects of Changes in Cardiorespiratory Fitness and Body Mass Index on All-Cause and Cardiovascular Disease Mortality in Men. Circulation. 2011;124:2483-90.

[34] Chodzko-Zajko W.J, Proctor D.N, Fiatarone Singh MA, et al.American College of Sports Medicine Position Stand. Exercise and Physical Activity for Older Adults. Med Sci Sport Exerc 2009;41:1510-30.

[35] Talbot LA, Gaines JM, Huynh TN, et al. A Home-Based Pedometer-Driven Walking Program to Increase Physical Activity in Older Adults with Osteoarthritis of the Knee: A Preliminary Study. J Am Geriatr Soc 2003;51:387-92.

[36] Toda Y, Toda T, Takemura S, et al. Change in Body Fat, but not Body Weight or Metabolic Correlates of Obesity, is Related to Symptomatic Relief of Obese Patients with Knee Osteoarthritis after a Weight Control Program. J Rheumatol 1998;25:2181-6. 